\title{
Anchor effect limits
}

ROBERT ADAMSON

FLORIDA ATLANTIC UNIVERSITY

In a category rating study, anchors of $.01 \mathrm{sec}, .05 \mathrm{sec}$, and $1.0 \mathrm{sec}$ were compared as to their effects on judgments of duration for series stimuli of $2,4,6,8$, and 10 sec. The rationale was to try to find a breakpoint in terms of anchor effects; Le., an extreme stimulus value which did not affect series judgments. Since the .01 sec anchor had no effect in lowering adaptation level, whereas both of the other anchors did $11 \mathrm{sec}$ group, $p=.05 ; .05$ sec group, $p=.03)$, the assumption of a breakpoint was supported.

Bevan \& Pritchard (1963) have conceptualized stimulus relevance in terms of anchor effects (AE), that is, the modification of judgments made about a series of stimuli as the result of exposing the subject to stimulus values which fall outside of the series. Essentially, the argument is that if exposure to a particular stimulus affects reactions to another stimulus, then the stimuli are relevant to each other. AE have been shown for a large number of intensitive dimensions, and are of particular interest because they enable detection of influences on the organism of minute physical intensities or intensity differences, even subliminal anchors modifying judgments of supraliminal stimull (Black \& Bevan, 1960; Bevan \& Pritchard, 1963a).

If relevance is defined in terms of AE, then somewhere on the stimulus dimension there should be a limiting anchor value for any given set of series stimuli (SS). Such a value would constitute one extreme of the relevance range for the $S S$, and values beyond it should no longer influence SS judgments. Accordingly, the purpose of the present study was to explore the influence of different anchor stimuli, successively more disparate from a set of SS, upon judgments made about the latter.

\section{Moftuod}

Twenty-six S8, Juniors and seniors at Florida Atlantic University, judged durations of bursts of white noise, at $74 \mathrm{~dB}$. The durations were $2,4,6,8$, and 10 sec, presented randomly over a series of 100 trials, at $8 \mathrm{sec}$ intervals. The test trials were preceded by a set of 20 practice trials. Ss were instructed to make judgments on a 9-point scale, ranging from "very, very short" to "very, very long." For the control group $(N=5)$, no anchor was presented. Three test groups received anchor stimuli of $.01 \sec (\mathrm{N}=8)$, $.05 \sec (N=5)$, and $1 \sec (N=8)$, respectively. Each anchor was a pure tone of $3000 \mathrm{cps}$, at $84 \mathrm{~dB}$, sounded $1 \mathrm{sec}$ before the appearance of each SS. Ss were told that the short tones would signal the subsequent burst of noise. Thus, they did not judge the anchors.

Control of stimulus presentation was by means of a program tape, one channel of which pulsed a Hunter timer set for the anchor duration. Another channel controlled a Hunter timer set for the SS duration. Each timer closed a speaker lead from, respectively, an oscillator and a white noise generator.

\section{Results}

Figure 1 shows the relation between judgments of duration and actual durations for the four groups. It appears that the anchor effect was greatest for the $.05 \mathrm{sec}$ group, next for the $1 \mathrm{sec}$ group, and nonexistent for the .01 sec group. Conversion of individual scores into adaptation levels (ALs, l.e., those stimuli values which correspond to a judgment of "medium") yields the following values: Control group, $A L=6 \mathrm{sec}$; .01 sec group, $\mathrm{AL}=5.81 \mathrm{sec} ; 1 \mathrm{sec}$ group, $\mathrm{AL}=\mathbf{5 . 2 6}$ sec; $.05 \mathrm{sec}$ group, $A L=4.88 \mathrm{sec}$.

Median comparisons of the ALs indicated that both the $.05 \mathrm{sec}$ and $1 \mathrm{sec}$ group differed significantly from the control $(p=.03, .05)$, whereas the .01 sec anchor group did not $(p=.43)$. The Fisher exact probability test was used for the comparisons (Fisher, 1936).

Further analysis indicated that the major effect of the anchor stimulus was limited to judgments of the 4 and $6 \mathrm{sec}$ intervals. When the $1 \mathrm{sec}$ and $.05 \mathrm{sec}$ groups were combined and compared to the combined control and .01 sec groups, the differences between median judgments for the $4 \mathrm{sec}$ stimulus is significant $(p=.009)$, as is the difference for the $6 \mathrm{sec}$ duration $(p=.011)$. Other differences were not significant.



Fig. 1. Category rating curves for a no anchor group (control) and three anchor groups as shown above. 


\section{Discussion}

The results support the idea that anchor stimuli are effective for a limited range on a stimulus continuum. A further inference is that such a range defines an area of relevance and that a stimulus dimension may be divided into a number of relevance subdimensions, each defined by limiting anchor effects. Additional work is being undertaken to check this assumption, since the present study treats only a restricted portion of the temporal continuum.

Differences between the anchor groups and the control for the 8 and 10 sec durations are not statistically significant, and it cannot be assumed that the $\mathrm{AE}$ held for these values. Thus the general finding (e.g., Helson, 1948; Michels \& Helson, 1949) that AE curves are negatively accelerated is not contraindicated.

The apparent, but not statistically significant superiority of the .05 anchor suggests the possibility that, as anchor stimuli deviate from the series, their effects may increase to a point, then show diminution. This possibility will be checked in subsequent studies.

\section{References}

BEVAN, W., \& PRITCHARD, J. F. The effect of subliminal tones upon the judgment of loudness. J. exp. Psychol., 1963a, 66, 23-29.

BEVAN, W., \& PRITCHARD, J. F. The anchor effect and the problem of relevance in the judgment of shape. J. gen Psychol, 1963b, 69, 147-161.

BLACK, R. W., \& BEVAN, W. The effect of subliminal shock upon the judged intensity of weak shock. Amer. J. Psychol, 1960, 73, 262-267.

HELSON, H. Adaptation-level as a basis for a quantitative theory of frame of reference. Psychol. Rev., 1948, 55, 297-313.

MICHELS, W. C., \& HELSON, H. A reformulation of the Fechner law in terms of adaptation-level applied to rating scale data. Amer. $J$. Psychol., 1949, 62, 355-368.

Note

1. This research was supported by the Air Force Office of Scientific Research, Grant No. 1163-66. 